\title{
EIGENVALUE INEQUALITIES AND MINIMAL SUBMANIFOLDS
}

\author{
ZHEN-RONG ZHOU
}

\begin{abstract}
Let $\left(S^{m}, g_{0}\right)$ be the unit sphere, $\left(M^{n}, g\right)$ its submanifold, $\lambda_{1}$ the first nonzero eigenvalue of $\left(M^{n}, g\right), H$ the mean curvature vector field of $M^{n}$. By Takahashi theorem, if $M^{n}$ is minimal, then $\lambda_{1} \leqq n$. In this paper, we establish some eigenvalue inequalities and use them to prove:

1. If $x$ is mass symmetric and of order $\{k, k+1\}$ for some $k$ such that $\lambda_{k} \geqq n$ or $\lambda_{k+1} \leqq n$, then $\varphi$ is minimal and $\lambda_{k}=n$ or $\lambda_{k+1}=n$.

2. If $H$ is parallel, $\int_{M} H d v_{M}=0$ and $\sigma^{2} \leqq \lambda_{1}$, then $H=0$ or $\sigma^{2}=\lambda_{1}$.

3. If $H$ is parallel and $\lambda_{k}=n$ for some $k$, then $H=0$ or $\sigma^{2}(x) \geqq \lambda_{k+1}-\lambda_{k}$ for some $x \in M^{n}$.

4. $\lambda_{1} \leqq \frac{n V^{2}}{V^{2}-\left(\int_{M} H d v_{M}\right)^{2}}$. Especially, if $\int_{M} H d v_{M}=0$, then $\lambda_{1} \leqq n$, and that $\lambda_{1}=n$ implies that $\varphi$ is minimal.
\end{abstract}

\section{Introduction}

Let $M^{n}$ be a compact $n$-dimensional Riemannian manifold, $\Delta_{M}$ the LaplaceBeltrami operator on $M^{n}$. Then $-\Delta_{M}$ has the discrete spectrum :

$$
\operatorname{spec}\left(\Delta_{M}\right)=\left\{0=\lambda_{0}<\lambda_{1}<\lambda_{2}<\cdots \rightarrow \infty\right\}
$$

Denote the unit hypersphere in the Euclidean space $E^{m+1}$ by $S^{m}$. A. Ros in [2] imbedded $S^{m}$ into the space of real symmetric matrices and obtained some eigenvalue inequalities for minimal submanifolds of $S^{m}$. In this paper, we consider more simple and more natural immersion $x: M^{n} \stackrel{\varphi}{\rightarrow} S^{m} \stackrel{\imath}{\rightarrow} E^{m+1}$, where $\varphi$ is an isometric immersion, not necessarily minimal, $i$ is the inclusion, and we also get some similar inequalities. Last, we use them to prove the following results:

Keywords. Immersion of $k$-type; order of an immersion; mean curvature vector (field); Weingarten transformation

Mathematical Subject Classifications (1991) 58G25

Received January 20, 1997; revised May 8, 1997. 
1. If $x$ is mass symmetric and of order $\{k, k+1\}$ for some $k$ such that $\lambda_{k} \geqq n$ or $\lambda_{k+1} \leqq n$, then $\varphi$ is minimal and $\lambda_{k}=n$ or $\lambda_{k+1}=n$.

2. If $H$ is parallel, $\int_{M} H d v_{M}=0$ and $\sigma^{2} \leqq \lambda_{1}$, then $H=0$ or $\sigma^{2}=\lambda_{1}$.

3. If $H$ is parallel and $\lambda_{k}=n$ for some $k$, then $H=0$ or $\sigma^{2}(x) \geqq \lambda_{k+1}-\lambda_{k}$ for some $x \in M^{n}$.

4. $\lambda_{1} \leqq \frac{n V^{2}}{V^{2}-\left(\int_{M} H d v_{M}\right)^{2}}$. Especially, if $\int_{M} H d v_{M}=0$, then $\lambda_{1} \leqq n$, and that $\lambda_{1}=n$ implies that $\varphi$ is minimal.

Here and below, $V$ is the volume of $M^{n}, H \in E^{m+1}$ the mean curvature vector field of $M^{n}$ in $S^{m}, \lambda_{1}$ the first nonzero eigenvalue of $\Delta_{M}, \sigma$ the length of the second fundamental form of $M^{n}$ in $S^{m}$; equalities for vectors mean that components of both ends equate, and integrations of vectors stand for those of components.

\section{Lemmas}

Let $x=i \circ \varphi$ be as above and $\langle$,$\rangle be the Euclidean inner product. From$ now on, we use $\Delta_{M}$ denote the Laplacian acting on functions over $M^{n}$ as well as on vector fields of $E^{m+1}$ which are restricted to $M^{n}$. In latter case, it acts on components. Then $x$ has $L^{2}$ decomposition: $x=x_{0}+\Sigma_{u \geqq 1} x_{u}$, where $x_{0}$ is a constant vector, $\Delta_{M} x_{u}=-\lambda_{u} x_{u} ; \int_{M}\left\langle x_{u}, x_{v}\right\rangle d v_{M}=\left\{\begin{array}{l}0 \quad u \neq v, \\ a_{u}(\geqq 0) \quad u=v .\end{array} \quad x_{0}\right.$ is called mass center of $x$. If $x_{0}$ is congrucent with the geometric center of $S^{m}$, we say that $x$ is mass-symmetric. If $\exists u_{\imath} \geqq 1, i=1, \ldots, k$, such that $x=x_{0}+\sum_{l=1}^{k} x_{u_{i}}$, then $x$ is called of $k$-type and $\left\{u_{1}, \ldots, u_{k}\right\}$ is by definition the order of $x$. It is known that if $\varphi$ is minimal, then $\varphi$ is mass symmetric, of 1-type and its order is $\{k\}$ for some $k$ by Takahashi theorem and Lemma 2.4 below. From the decomposition of $x$ we have:

$$
\Delta_{M} x=-\sum_{u \geq 0} \lambda_{u} x_{u} ; \Delta_{M}^{2} x=\sum_{u \geq 0} \lambda_{u}^{2} x_{u} .
$$

Define :

$$
\begin{gathered}
\Psi_{k}=-\int_{M}\left\langle\Delta_{M} x, x\right\rangle d v_{M}-\lambda_{k} \int_{M}\langle x, x\rangle d v_{M} \\
\Theta_{k}=\int_{M}\left\langle\Delta_{M} x, \Delta_{M} x\right\rangle d v_{M}+\lambda_{k} \int\left\langle\Delta_{M} x, x\right\rangle d v_{M} \\
\Omega_{k}=-\int_{M}\left\langle\Delta_{M}^{2} x, \Delta_{M} x\right\rangle d v_{M}-\lambda_{k} \int_{M}\left\langle\Delta_{M} x, \Delta_{M} x\right\rangle d v_{M}
\end{gathered}
$$

Then, we have 
LEMMA 2.1. For any integer $k \geqq 1$,

(i) $\Theta_{k}-\lambda_{k+1} \Psi_{k}=\sum_{u \geqq 0}\left(\lambda_{u}-\lambda_{k}\right)\left(\lambda_{u}-\lambda_{k+1}\right) a_{u} \geqq 0$

and the equality holds iff $x$ mass symmetric and is of order $\{k, k+1\}$;

(ii) $\Omega_{k}-\lambda_{k+1} \Theta_{k}=\sum_{u \geq 0} \lambda_{u}\left(\lambda_{u}-\lambda_{k}\right)\left(\lambda_{u}-\lambda_{k+1}\right) a_{u} \geqq 0$

and the equality holds iff $x$ is of order $\{k, k+1\}$.

Remark. The two inequalities above holds for $k \geqq 0$.

Let $H$ be the mean curvature vector field of $M^{n}$. Then Takahashi theorem says that $\Delta_{M} x=n H-n x$. Hence $\Delta_{M}^{2} x=n \Delta_{M} H-n^{2} H+n^{2} x$. Therefore,

$$
\begin{gathered}
\int_{M}\langle x, x\rangle d v_{M}=V ; \int_{M}\left\langle\Delta_{M} x, x\right\rangle d v_{M}=-n V ; \\
\int_{M}\left\langle\Delta_{M} x, \Delta_{M} x\right\rangle d v_{M}=n^{2} \int_{M}\langle H, H\rangle d v_{M}+n^{2} V ; \\
\int_{M}\left\langle\Delta_{M}^{2} x, \Delta_{M} x\right\rangle d v_{M}=n^{2} \int_{M}\left\langle\Delta_{M} H, H\right\rangle d v_{M}-2 n^{3} \int_{M}\langle H, H\rangle d v_{M}-n^{3} V ; \\
\Psi_{k}=\left(n-\lambda_{k}\right) V ; \Theta_{k}=n^{2} \int_{M}\langle H, H\rangle d v_{M}+n\left(n-\lambda_{k}\right) V ; \\
\Omega_{k}=-n^{2} \int_{M}\left\langle\Delta_{M} H, H\right\rangle d v_{M}+n^{2}\left(2 n-\lambda_{k}\right) \int_{M}\langle H, H\rangle d v_{M}+n^{2}\left(n-\lambda_{k}\right) V .
\end{gathered}
$$

From these formulas, we have:

LEMMA 2.2. For any $k \geqq 0$,

(i) $\Theta_{k}-\lambda_{k+1} \Psi_{k}=n^{2} \int_{M} H^{2} d v_{M}+\left(n-\lambda_{k}\right)\left(n-\lambda_{k+1}\right) V$,

(ii) $\Omega_{k}-\lambda_{k+1} \Theta_{k}=-n^{2} \int_{M}\left\langle\Delta_{M} H, H\right\rangle d v_{M}+n^{2}\left(2 n-\lambda_{k}-\lambda_{k+1}\right) \int_{M} H^{2} d v_{M}$

$$
+n\left(n-\lambda_{k}\right)\left(n-\lambda_{k+1}\right) V \text {. }
$$

Next, use $\nabla^{\perp}$ to denote the normal connection of $\varphi, \sigma$ the length of the second fundamental form of $\varphi$. We have

LEMMA 2.3.

$$
-\int_{M}\left\langle\Delta_{M} H, H\right\rangle d v_{M} \leqq \int_{M}\left\langle\nabla^{\perp} H, \nabla^{\perp} H\right\rangle d v_{M}+\int_{M} H^{2} \sigma^{2} d v_{M}
$$

where the equality holds whenever the codimension is one. 
Proof. Without loss of generality, we let $M^{n} \subset S^{m}$, and $\varphi=i$, the inclusion. Let $\nabla$ and $\bar{\nabla}$ be the Riemannian connections of $M^{n}$ and $S^{m}$ respectively. On $M^{n}$, we take a local field of orthonormal frame: $e_{1}, \ldots, e_{n}$. Then by Gauss and Weingarten formulas we get:

$$
\begin{aligned}
\frac{1}{2} \Delta_{M}\langle H, H\rangle & =\frac{1}{2} \sum_{j=1}^{n} \nabla_{e_{j}} \nabla_{e_{j}}\langle H, H\rangle=\frac{1}{2} \sum_{j=1}^{n} \nabla_{e_{j}}\left(\nabla_{e_{j}}\langle H, H\rangle\right) \\
& =\sum_{j=1}^{n} \nabla_{e_{j}}\left\langle\bar{\nabla}_{e_{j}} H, H\right\rangle=\sum_{j=1}^{n} \nabla_{e_{j}}\left\langle\nabla_{e_{j}}^{\perp} H, H\right\rangle \\
& =\sum_{j=1}^{n} \bar{\nabla}_{e_{j}}\left\langle\nabla_{e_{j}}^{\perp} H, H\right\rangle \\
& =\sum_{j=1}^{n}\left(\left\langle\bar{\nabla}_{e_{j}}\left(\nabla_{e_{j}}^{\perp} H\right), H\right\rangle+\left\langle\nabla_{e_{j}}^{\perp} H, \nabla_{e_{j}} H\right\rangle\right) \\
& =\sum_{j=1}^{n}\left\langle\nabla_{e_{j}}^{\perp} \nabla_{e_{j}}^{\perp} H, H\right\rangle+\sum_{j=1}^{n}\left\langle\nabla_{e_{j}}^{\perp} H, \nabla_{e_{j}}^{\perp} H\right\rangle \\
& \triangleq\left\langle\Delta^{\perp} H, H\right\rangle+\left\langle\nabla^{\perp} H, \nabla^{\perp} H\right\rangle
\end{aligned}
$$

Define $\Delta_{M} v=\sum_{j=1}^{n}\left(D_{e_{j}} D_{e_{j}} v-D_{\nabla_{e_{j} e_{j}}} v\right)$, where $D$ is the flat connection of $E^{m+1}$, $v=v(x) \in E^{m+1}, n \in M^{n}$. It is easy to verify that $\Delta_{M}$ defined here is exactly the Laplacian acting on vectors by on their components which is defined at the begining of this section. We can choose $\left\{e_{\imath}, i=1,2, \ldots, n\right\}$ such that $\nabla_{e_{i}} e_{j}=0$. Hence we have

$$
\begin{aligned}
\Delta_{M} H & =\sum_{j=1}^{n} D_{e_{j}} D_{e_{j}} H=\sum_{j=1}^{n} D_{e_{j}}\left(\bar{\nabla}_{e_{j}} H+\bar{B}\left(e_{\jmath}, H\right)\right) \\
& =\sum_{j=1}^{n} D_{e_{j}} \bar{\nabla}_{e_{j}} H \quad \text { because } S^{n} \text { is totally umbilical } \\
& =\sum_{j=1}^{n}\left[\bar{\nabla}_{e_{j}} \bar{\nabla}_{e_{j}} H+\bar{B}\left(e_{\jmath}, \bar{\nabla}_{e_{j}} H\right)\right]
\end{aligned}
$$

where, $\bar{B}$ and $\bar{A}$ are the second fundamental form and Weingarten transformation of $S^{m}$ respectively. In the following, we use $B$ and $A$ stand for corresponding quantities of $M^{n}$ in $S^{m}$. Then

$$
\begin{aligned}
& \sum_{j=1}^{n} \bar{\nabla}_{e_{j}}\left(\bar{\nabla}_{e_{j}} H\right)=\sum_{j=1}^{n} \bar{\nabla}_{e_{j}}\left(-A_{H} e_{j}+\nabla_{e_{j}}^{\perp} H\right) \\
& =\sum_{j=1}^{n}-\left(\nabla_{e_{j}}\left(A_{H} e_{\jmath}\right)+B\left(A_{H} e_{\jmath}, e_{j}\right)\right)+\sum_{j=1}^{n}\left(-A_{\nabla_{e_{j}}^{\perp} H} e_{j}+\nabla_{e_{j}}^{\perp} \nabla_{e_{j}}^{\perp} H\right) \\
& =-\sum_{j=1}^{n}\left[\nabla_{e_{j}}\left(A_{H} e_{\jmath}\right)+A_{\nabla_{e_{j}}^{\perp} H} e_{\jmath}\right]+\sum_{j=1}^{n}\left(-B\left(A_{H} e_{\jmath}, e_{\jmath}\right)+\nabla_{e_{j}}^{\perp} \nabla_{e_{j}}^{\perp} H\right) .
\end{aligned}
$$

From the above calculations, we have 


$$
\begin{aligned}
\left\langle\Delta_{M} H, H\right\rangle & =-\sum_{\jmath=1}^{n}\left\langle B\left(A_{H} e_{\jmath}, e_{j}\right), H\right\rangle+\left\langle\Delta^{\perp} H, H\right\rangle \\
& =-\sum_{\jmath=1}^{n}\left\langle A_{H} e_{\jmath}, A_{H} e_{\jmath}\right\rangle+\left\langle\Delta^{\perp} H, H\right\rangle \\
& \triangleq-\left\langle A_{H}, A_{H}\right\rangle+\left\langle\Delta^{\perp} H, H\right\rangle
\end{aligned}
$$

where $\Delta^{\perp}=\sum_{\jmath=1}^{n} \nabla_{e_{j}}^{\perp} \nabla_{e_{j}}^{\perp}$. So $\left\langle\Delta^{\perp} H, H\right\rangle=\left\langle\Delta_{M} H, H\right\rangle+\left\langle A_{H}, A_{H}\right\rangle$. Hence we have

$$
\frac{1}{2} \Delta_{M}\langle H, H\rangle=\left\langle\Delta_{M} H, H\right\rangle+\left\langle\nabla^{\perp} H, \nabla^{\perp} H\right\rangle+\left\langle A_{H}, A_{H}\right\rangle .
$$

Therefore,

$$
-\int_{M}\left\langle\Delta_{M} H, H\right\rangle d v_{M}=\int_{M}\left\langle\nabla^{\perp} H, \nabla^{\perp} H\right\rangle d v_{M}+\int_{M}\left\langle A_{H}, A_{H}\right\rangle d v_{M} .
$$

Take a local unit normal vector field $e_{n+1}$ of $M^{n}$ in $T S^{m}$ such that $e_{n+1}$ is parallel with $H$, i.e. $H=|H| e_{n+1}$. Then

$$
\begin{aligned}
\left\langle A_{H}, A_{H}\right\rangle & =\sum_{j=1}^{n}\left\langle A_{H} e_{\jmath}, A_{H} e_{\jmath}\right\rangle=H^{2} \sum_{\jmath=1}^{n}\left\langle A_{n_{+1}} e_{\jmath}, A_{n_{+1}} e_{\jmath}\right\rangle \\
& =H^{2}\left\|A_{n+1}\right\|^{2} \leqq H^{2} \sigma^{2}
\end{aligned}
$$

and equality holds if the codimension of $M^{n}$ in $S^{m}$ is one. Now, the proof of Lemma 2.3 is complete.

In next section, when we discuss the upper bound of $\lambda_{1}$, we need the following two lemmas.

LEMMA 2.4. $M^{n}$ is mass symmetric iff $\int_{M} H d v_{M}=0$.

Proof. By Takahashi theorem and the $L^{2}$ decompositions of $x$ and $H$, we have

$$
\Delta x=n(H-x)=n\left(H_{0}-x_{0}\right)+\sum_{u \geq 1} n\left(H_{u}-x_{u}\right) .
$$

Integrating both ends and noting that when $u \geqq 1, \int_{M} H_{u} d v_{M}=\int_{M} x_{u} d v_{M}=0$, we can obtain: $\int_{M} H_{0} d v_{M}=\int_{M} x_{0} d v_{M}$, i.e. $H_{0}=x_{0}$. Hence $\int_{M} H d v_{M}=\int_{M} H_{0} d v_{M}=$ $\int_{M} x_{0} d v_{M}=x_{0} V$. Lemma 2.4 follows.

LEMMA 2.5. $\left(\int_{M} H d v_{M}\right)^{2} \leqq V^{2}$, and the equality is true iff $x$ is constant.

Proof. $\left(\int_{M} H d v_{M}\right)^{2}=\left(\int_{M} H_{0} d v_{M}\right)^{2}=\left(\int_{M} x_{0} d v_{M}\right)^{2}=\left\langle x_{0}, x_{0}\right\rangle V^{2}$

$$
=\int_{M}\left\langle x_{0}, x_{0}\right\rangle d v_{M} \cdot V \leqq \int_{M}\langle x, x\rangle d v_{M} \cdot V=V^{2},
$$

and the equality holds iff $x=x_{0}$. 


\section{Eigenvalue inequalities and their corollaries}

By Takahashi theorem, if $\varphi$ is minimal, then $\varphi$ is mass symmetric and of order $\{k\}$ for some $k$, and $\lambda_{k}=n$. The following theorem shows that the inverse is true.

THEOREM 3.1. If $i \circ \varphi$ is mass symmetric and of order $\{k, k+1\}$ for some $k$ such that $\lambda_{k} \geqq n$ or $\lambda_{k+1} \leqq n$, then $\varphi$ is minimal (hence is of 1-type by Takahashi theorem) and $\lambda_{k}=n$ or $\lambda_{k+1}=n$.

Proof. By Lemmas 2.1-(i) and 2.2-(i) we have

$$
n^{2} \int_{M} H^{2} d v_{M}+\left(n-\lambda_{k}\right)\left(n-\lambda_{k+1}\right) V=0
$$

from which the result follows.

THEOREM 3.2. For any $k \geqq 0$

$$
\begin{gathered}
n \int_{M}\left\langle\nabla^{\perp} H, \nabla^{\perp} H\right\rangle d v_{M}+n \int_{M} H^{2}\left(\sigma^{2}+2 n-\lambda_{k}-\lambda_{k+1}\right) d v_{M} \\
+\left(n-\lambda_{k}\right)\left(n-\lambda_{k+1}\right) V \geqq 0
\end{gathered}
$$

where the equality for $k \geqq 1$ implies that $\varphi$ is of order $\{k, k+1\}$.

Proof. From Lemmas 2.1-(ii), 2.2-(ii) and 2.3 we can reach the inequality we need. The equality shows that $i \circ \varphi$ is of order $\{k, k+1\}$ by Lemma 2.1-(ii).

COROLlARY 3.1. Let $\varphi$ have parallel mean curvature normal and $\lambda_{k}=n$ for some $k$. Then $M^{n}$ is minimal or $\sigma^{2}(x) \geqq \lambda_{k+1}-\lambda_{k}$ for some $x \in M^{n}$.

THEOREM 3.3. Let $x_{0}$ be the mass center of $x=i \circ \varphi$, then for any real number $t$, we have

$$
\begin{aligned}
\left(n-\lambda_{1}\right)(n-t)^{2} V+2 n^{2}(n-t) \int_{M} H^{2} d v_{M}+n^{2} \int_{M}\left\langle\nabla^{\perp} H, \nabla^{\perp} H\right\rangle d v_{M} \\
\quad+n^{2} \int_{M} H^{2}\left(\sigma^{2}-\lambda_{1}\right) d v_{M}+\lambda_{1} t^{2} \int_{M}\left\langle x_{0}, x_{0}\right\rangle d v_{M} \geqq 0 .
\end{aligned}
$$

Proof. Set $F_{t}=-\Delta_{M} x-t\left(x-x_{0}\right)$. Then $\int_{M} F_{t} d v_{M}=0$. By the minimal principle for $\lambda_{1}$ we have

$$
-\int_{M}\left\langle\Delta_{M} F_{t}, F_{t}\right\rangle d v_{M} \geqq \lambda_{1} \int_{M}\left\langle F_{t}, F_{t}\right\rangle d v_{M} .
$$

On the other hand, 


$$
\begin{gathered}
F_{t}=-n H+n x-t\left(x-x_{0}\right), \quad \Delta_{M} F_{t}=-n \Delta_{M} H+n(n-t)(H-x), \\
\int_{M}\left\langle x, x_{0}\right\rangle d v_{M}=\int_{M}\left\langle x_{0}, x_{0}\right\rangle d v_{M}, \int_{M}\left\langle\Delta_{M} x, x_{0}\right\rangle d v_{M}=0, \\
\int_{M}\left\langle\Delta_{M} H, x_{0}\right\rangle d v_{M}=0, \int_{M}\left\langle H, x_{0}\right\rangle d v_{M}=\int_{M}\left\langle x_{0}, x_{0}\right\rangle d v_{M} .
\end{gathered}
$$

Therefore, we reach

$$
\begin{gathered}
\int_{M}\left\langle\Delta_{M} F_{t}, F_{t}\right\rangle d v_{M}=n^{2} \int_{M}\left\langle\Delta_{M} H, H\right\rangle d v_{M}-2 n^{2}(n-t) \int_{M} H^{2} d v_{M}-n(n-t)^{2} V, \\
\int_{M}\left\langle F_{t}, F_{t}\right\rangle d v_{M}=n^{2} \int_{M} H^{2} d v_{M}+(n-t)^{2} V-t^{2} \int_{M}\left\langle x_{0}, x_{0}\right\rangle d v_{M} .
\end{gathered}
$$

Substituting the last two equalities into (*) and using Lemma 2.3, the theorem follows.

For minimal submanifolds of a unit sphere, if $\sigma^{2} \leqq n$, then $\sigma^{2}=0$ or $n$. For non-minimal ones, we also have a similar result (see (ii) below).

COROLlary 3.2. Suppose that $x=i \circ \varphi$ is mass symmetric or equivalently that $\int_{M} H d v_{M}=0$

(i) If $\lambda_{1}=n$, then $\varphi$ is minimal.

(ii) If $\nabla^{\perp} H=0, \sigma^{2} \leqq \lambda_{1}$, then $\sigma^{2}=\lambda_{1}$ unless $H=0$.

Proof. Under the assumption of (i), using Theorem 3.3 (taking $t>n$ ), we have

$$
\int_{M} H^{2} d v_{M} \leqq \frac{1}{2(n-t)}\left\{-\int_{M}\left\langle\nabla^{\perp} H, \nabla^{\perp} H\right\rangle d v_{M}-\int_{M} H^{2}\left(\boldsymbol{\sigma}^{2}-\lambda_{1}\right) d v_{M}\right\}
$$

Let $t \rightarrow \infty$ we have $H=0$.

For the proof of (ii), we use Theorem 3.3 (take $t=n$ ) and Lemma 2.4. We get $H^{2} \int_{M}\left(\sigma^{2}-\lambda_{1}\right) d v_{M} \geqq 0$. Then the (ii) follows.

COROLlaRy 3.3. $\lambda_{1} \leqq \frac{n V^{2}}{V^{2}-\left(\int_{M} H d v_{M}\right)^{2}}$.

Especially, if $\int_{M} H d v_{M}=0$, then $\lambda_{1} \leqq n$. Furthermore, $\lambda_{1}=n$ implies $H=0$.

Proof. Let the both ends of the inequality in Theorem 3.3 be divided by $(n-t)^{2}$, then let $t$ go to infinity. The inequality is obtained. The rest follows from Corollary 3.2-(i).

Remark. Let $M$ be an $n$-dimensional compact submanifold of the unit hypersphere $S^{m}$ of an Euclidean $m+1$-space with lower order $p$ and upper order q. B. Y. Chen proved the following two statements (see [1] p. 144 Corollary 6.13): 
(1) If $M$ is mass symmetric, then $\lambda_{1} \leqq \lambda_{p} \leqq n$. In particular, $\lambda_{p}=n$ iff $M$ is of 1-type and of order $\{p\}$.

(2) If $M$ is of finite type, then $\lambda_{q} \geqq n$. In particular, $\lambda_{q}=n$ iff $M$ is of 1 type and of order $\{q\}$.

Because 1-type is 2-type, by Theorem 3.1 we know that $M$ in (1) and (2) above is in fact minimal (if $M$ is also of mass symmetric in (2)).

\section{REFERENCES}

[1] B. Y. Chen, A report on submanifolds of finite type, Soochow J. Math., 22 (1996), 117-337.

[2] A. Ros, Eigenvalue inequalities for minimal submanifolds and $P$-manifolds, Math. Z., 187 (1984), 393-404.

[3] Y.B. SHen, On eigenvalue inequalities of minimal and Kaehlerian submanifolds, Acta Math. Sinica A, 34 (1991), 551-560 (in Chinese).

[4] T. Takahashi, Minimal immersions of Riemannian manifolds, J. Math. Soc. Japan, 18 (1966), 380-385.

WUHAN UNIVERSITY

430072, WUHAN

P.R. CHINA 